\title{
Severe Liver Injury Associated with Glecaprevir Plus Pibrentasvir Therapy in a Patient with Treatment-naïve Hepatitis C Virus Infection
}

\author{
Tasuku Hara ${ }^{1}$, Tomoya Ohara ${ }^{1}$, Masashi Taniguchi ${ }^{1}$, Hiroaki Sakai ${ }^{1}$, Kohei Oka ${ }^{1}$, \\ Naoto Iwai ${ }^{1}$, Toshifumi Tsuji ${ }^{1}$, Takashi Okuda ${ }^{1}$, Akihiro Nagata ${ }^{2}$, Toshiyuki Komaki ${ }^{1}$, \\ Junichi Sakagami ${ }^{1}$ and Keizo Kagawa ${ }^{1}$
}

\begin{abstract}
:
A 49-year-old man underwent treatment with glecaprevir plus pibrentasvir (G/P) for chronic hepatitis $\mathrm{C}$ infection. Six weeks later, he was admitted to our hospital because of jaundice and fatigue with no accompanying skin rash. A laboratory examination and evaluation of the patient's history resulted in a diagnosis of acute liver injury. Discontinuation of G/P and a rigorous medical protocol, including plasma exchange and hemodiafiltration, successfully mitigated the liver damage. The patient was also found to be allergic to two drugs other than the G/P therapy. In such cases with a history of drug allergy, careful observation may be required to detect serious adverse events.
\end{abstract}

Key words: chronic hepatitis C, glecaprevir plus pibrentasvir, liver injury, side effect, drug allergy

(Intern Med 60: 2437-2443, 2021)

(DOI: 10.2169/internalmedicine.6664-20)

\section{Introduction}

Hepatitis C virus (HCV) is a leading cause of chronic hepatitis, cirrhosis, and hepatocellular carcinoma (1). With the introduction of direct-acting antiviral agents (DAAs), the efficacy and safety of the treatment of chronic hepatitis $\mathrm{C}$ infection has improved significantly (2). Glecaprevir [nonstructural protein 3/4A (NS3/4A) protease inhibitor] plus pibrentasvir [nonstructural protein 5A (NS5A) inhibitor] (G/P) therapy comprises ribavirin-free treatment with a DAA and has the advantage of a shorter treatment duration than other regimens; furthermore, the treatment is pan-genotypic and thus recommended for all genotypes of HCV infection (3).

$\mathrm{G} / \mathrm{P}$ has been reported to exhibit a strong antiviral effect with a good safety profile and a low rate of side effects (46). However, we observed one instance of severe liver injury that occurred during the administration of $\mathrm{G} / \mathrm{P}$ in a patient with treatment-naïve genotype $1 \mathrm{~b} \mathrm{HCV}$ infection. The indi- vidual risk of drug-induced liver injury (DILI) and its associated clinical phenotype are likely to be determined by the complex interplay between the physiochemical and toxicological properties of drugs, host factors, and the resulting interactions between them (7).

We herein report a rare case of an adverse event caused by interplay between G/P therapy and host risk factors.

\section{Case Report}

The patient was a 49-year-old man (height: $175.0 \mathrm{~cm}$; weight: $77.2 \mathrm{~kg}$; body mass index: 25.2 ) with a tattoo and a history of allergy with isopropylantipyrine. He was first referred to our hospital due to liver dysfunction at the age of 48. He had underlying conditions of insomnia and reflux esophagitis, for which he had been taking etizolam, brotizolam, and esomeprazole.

His workup revealed co-infection with $\mathrm{HCV}$ (genotype 1 b, $5.8 \log \mathrm{IU} / \mathrm{mL}$ ) and hepatitis B virus (HBV) (genotype C,

${ }^{1}$ Department of Gastroenterology and Hepatology, Fukuchiyama City Hospital, Japan and ${ }^{2}$ Department of Pathology, Fukuchiyama City Hospital, Japan

Received: November 6, 2020; Accepted: January 4, 2021; Advance Publication by J-STAGE: February 22, 2021

Correspondence to Dr. Tasuku Hara, t-hara@koto.kpu-m.ac.jp 
Table 1. Laboratory Data at the Start of Glecaprevir Plus Pibrentasvir Therapy.

\begin{tabular}{|c|c|c|c|}
\hline Variable & & Variable & \\
\hline White blood cells $(/ \mu \mathrm{L})$ & 5,880 & Total protein $(\mathrm{g} / \mathrm{dL})$ & 7.3 \\
\hline Neutrophils (\%) & 50.0 & Albumin $(\mathrm{g} / \mathrm{dL})$ & 3.9 \\
\hline Eosinophils (\%) & 1.7 & Total bilirubin (mg/dL) & 1.1 \\
\hline Basophils (\%) & 0.7 & AST (IU/L) & 100 \\
\hline Monocytes (\%) & 11.4 & ALT (IU/L) & 51 \\
\hline Lymphocytes (\%) & 36.2 & LDH (IU/L) & 185 \\
\hline Red blood cells $\left(10^{4} / \mu \mathrm{L}\right)$ & 409 & ALP (IU/L) & 436 \\
\hline Hematocrit $(\%)$ & 41.4 & GGT (IU/L) & 125 \\
\hline Hemoglobin (g/dL) & 14.0 & BUN (mg/dL) & 10 \\
\hline Platelets $\left(10^{4} / \mu \mathrm{L}\right)$ & 19.2 & Creatinine (mg/dL) & 0.66 \\
\hline C-reactive protein $(\mathrm{mg} / \mathrm{dL})$ & 0.05 & & \\
\hline \multirow[t]{2}{*}{ Glucose $(\mathrm{mg} / \mathrm{dL})$} & 162 & HCV-RNA (log IU/mL) & 1.4 \\
\hline & & HBsAg (IU/mL) & 18.2 \\
\hline PT-INR & 1.21 & HBV-DNA $(\log \mathrm{IU} / \mathrm{mL})$ & negative \\
\hline PT $(\%)$ & 67.8 & HBcrAg $(\log \mathrm{U} / \mathrm{mL})$ & $\leq 2.9$ \\
\hline FIB-4 index & 3.57 & HBV genotype & $\mathrm{C}$ \\
\hline
\end{tabular}

PT: prothrombin time, PT-INR: prothrombin time-international normalized ratio, FIB-4 index: fibrosis-4 index, AST: aspartate aminotransferase, ALT: alanine aminotransferase, LDH: lactate dehydrogenase, ALP: alkaline phosphatase, GGT: gamma-glutamyl transpeptidase, BUN: blood urea nitrogen, HCV-RNA: hepatitis C virus RNA, HBsAg: hepatitis B surface antigen, HBV-DNA: hepatitis B virus DNA, HBcrAg: hepatitis B core-related antigen

quantity undetectable). Since the level of serum HBV DNA level was negative, and the level of hepatitis B surface antigen (HBs-Ag) was low (33.7 IU/mL), he was diagnosed as an inactive HBV carrier. Although the patient had stopped consuming alcohol, he had previously consumed ethanol equivalent to $60 \mathrm{~g}$ a day. Six months after he stopped, he started treatment with an 8-week course for $\mathrm{HCV}$ with 3 tablets of glecaprevir $(100 \mathrm{mg}) /$ pibrentasvir $(40 \mathrm{mg})$ once a day. The patient's laboratory data at the treatment initiation are shown in Table 1.

After 6 weeks of G/P, the patient complained of vomiting, abdominal pain, and jaundice. He visited our hospital 3 days later and was found to have severe liver injury with a total bilirubin (T-Bil) level of $20.6 \mathrm{mg} / \mathrm{dL}$. The patient's data with regard to laboratory parameters and antibodies to other possible viral infections are summarized in Table 2. HBV reactivation was excluded from the causes of liver injury, as the level of serum HBV DNA remained negative, and the level of HBs-Ag was low $(7.8 \mathrm{IU} / \mathrm{mL})$. Since there was no history of recent ingestion of other drugs or alcohol, we considered the possibility of DILI due to the G/P therapy, and G/P was immediately discontinued. During treatment with $\mathrm{G} / \mathrm{P}$, the patient had complained of fatigue, although he did not experience skin rash or a fever. The results of a drug-induced lymphocyte stimulation test (DLST) for G/P were negative. Based on the Digestive Disease Week Japan 2004 (DDW-J) scale (8), this type of liver damage was classified as cholestatic liver injury, and an association between G/P and liver injury was deemed possible (score of 4).

A physical examination on admission showed neither as- cites nor signs of hepatic encephalopathy. The clinical course is summarized in Fig. 1 (date of hospitalization was labeled as day 0). In addition, serial changes in liver function tests and viral markers are shown in Table 3. After hospitalization, the serum T-Bil level gradually improved without any treatment, but the prothrombin time (PT) level worsened. On day 9, the patient developed asterixis and was diagnosed with grade II hepatic encephalopathy. Although the observed PT $(54.7 \%)$ did not meet the criteria for acute liver failure (9), since the patient was developing hepatic encephalopathy, plasma exchange (PE) and hemodiafiltration (HDF) procedures were initiated along with oral treatment of lactulose and rifaximin. After three PE sessions, and one HDF session, his liver function improved, and he recovered from hepatic encephalopathy. Each PE session included the administration of 40 units of fresh-frozen plasma (FFP). One hour after initiating the first PE session, the patient developed an anaphylactic reaction (skin rashes with slight dyspnea), and was treated with methylprednisolone $(125 \mathrm{mg})$. Consequently, he was started on tenofovir alafenamide fumarate (TAF) to prevent $\mathrm{HBV}$ reactivation. However, no side effects were observed when FFP was administered on day 8 .

The patient developed a fever with a body temperature of $39^{\circ} \mathrm{C}$ on day 24 . The results of a subsequent catheter tip culture revealed growth of Staphylococcus capitis subsp. ureolyticus; therefore, we prioritized treatment for sepsis. Empirical antibiotic therapy with vancomycin was initiated, and it was de-escalated to cefazolin (CEZ) on day 32. However, drug eruption appeared 4 days after the switch to CEZ 
Table 2. Laboratory Data on Admission.

\begin{tabular}{|c|c|c|c|}
\hline \multirow{2}{*}{$\frac{\text { Variable }}{\text { White blood cells }(/ \mu \mathrm{L})}$} & \multicolumn{3}{|c|}{ Variable } \\
\hline & 10,260 & Total protein $(\mathrm{g} / \mathrm{dL})$ & 7.2 \\
\hline Neutrophils (\%) & 78.4 & Albumin $(\mathrm{g} / \mathrm{dL})$ & 3.6 \\
\hline Eosinophils (\%) & 0.5 & Total bilirubin (mg/dL) & 20.6 \\
\hline Basophils (\%) & 0.1 & Direct bilirubin $(\mathrm{mg} / \mathrm{dL})$ & 15.4 \\
\hline Monocytes (\%) & 7.0 & AST (IU/L) & 205 \\
\hline Lymphocytes (\%) & 14.0 & ALT (IU/L) & 65 \\
\hline Red blood cells $\left(10^{4} / \mu \mathrm{L}\right)$ & 372 & LDH (IU/L) & 246 \\
\hline Hematocrit $(\%)$ & 34.1 & ALP (IU/L) & 442 \\
\hline Hemoglobin (g/dL) & 12.3 & GGT (IU/L) & 124 \\
\hline Platelets $\left(10^{4} / \mu \mathrm{L}\right)$ & 11.7 & BUN (mg/dL) & 4 \\
\hline C-reactive protein $(\mathrm{mg} / \mathrm{dL})$ & 0.65 & Creatinine (mg/dL) & 0.88 \\
\hline Glucose $(\mathrm{mg} / \mathrm{dL})$ & 178 & $\mathrm{NH}_{3}(\mathrm{mg} / \mathrm{dL})$ & 55 \\
\hline \multirow{2}{*}{$\mathrm{HbA} 1 \mathrm{c}(\mathrm{N})(\%)$} & 6.8 & HCV-RNA (log IU/mL) & negative \\
\hline & & HBsAg (IU/mL) & 7.8 \\
\hline PT-INR & 1.21 & IgM anti-HBc & $(-)$ \\
\hline PT $(\%)$ & 67.8 & HBV-DNA (log IU/mL) & negative \\
\hline \multirow[t]{2}{*}{ FIB-4 index } & 10.65 & $\mathrm{HBeAg}$ & $(-)$ \\
\hline & & $\operatorname{HBeAb}(\%)$ & $(+)$ \\
\hline $\operatorname{IgG}(\mathrm{mg} / \mathrm{dL})$ & 1,462 & HBcrAg $(\log \mathrm{U} / \mathrm{mL})$ & $\leq 2.9$ \\
\hline $\operatorname{IgA}(\mathrm{mg} / \mathrm{dL})$ & 421 & IgM anti-HAV & $(-)$ \\
\hline $\operatorname{IgM}(\mathrm{mg} / \mathrm{dL})$ & 101 & IgA anti-HEV & $(-)$ \\
\hline $\operatorname{IgE}(\mathrm{IU} / \mathrm{mL})$ & 18 & IgM anti-EBV VCA & $(-)$ \\
\hline Anti-nuclear antibody & $<40$ & IgG anti-EBV VCA & $(+)$ \\
\hline Anti-mitochondria M2 & 1.4 & EBNA & $(-)$ \\
\hline Anti-smooth muscle & $<20$ & IgM anti-CMV & $(-)$ \\
\hline Anti-LKM1 & $<5.0$ & IgM anti-HSV & $(-)$ \\
\hline
\end{tabular}

PT: prothrombin time, PT-INR: prothrombin time-international normalized ratio, FIB-4 index: fibrosis-4 index, HbA1c $(\mathrm{N})$ : glycated hemoglobin, Ig: immunoglobulin, AntiLKM1: anti-liver-kidney microsome type 1 antibody, AST: aspartate aminotransferase, ALT: alanine aminotransferase, LDH: lactate dehydrogenase, ALP: alkaline phosphatase, GGT: gamma-glutamyl transpeptidase, BUN: blood urea nitrogen, HCV-RNA: hepatitis C virus RNA, HBsAg: hepatitis B surface antigen, anti-HBc: hepatitis B virus core antibody, HBV-DNA: hepatitis B virus DNA, HBeAg: hepatitis B envelope antigen, HBeAb: anti-HBe antibody, HBcrAg: hepatitis B core-related antigen, anti-HAV: anti-hepatitis A virus antibody, HEV: hepatitis E virus, anti-EBV VCA: anti-Epstein-Barr virus capsid antigen antibody, EBNA: Epstein-Barr virus nuclear antigen, CMV: cytomegalovirus, HSV: herpes simplex virus

(after approximately 2 weeks of antibiotic treatment), so treatment was discontinued. The drug eruption consequently resolved within a few days. The results of a DLST for CEZ were negative. On day 33, an ultrasound-guided percutaneous liver biopsy was performed. Subsequently, on day 42, the patient was discharged from the hospital. At this point, 12 weeks after the end of G/P, HCV RNA was not detected.

\section{Histological findings of the liver biopsy}

The biopsy specimen revealed cross-linked fibrosis between the portal veins, and lymphocyte and plasma cell infiltrate in the portal vein area, with slight interface hepatitis observed. There was no noticeable liver steatosis. Ductular proliferation, ballooning hepatocytes, and cholestasis, which are consistent with DILI, were observed (Fig. 2). Although a liver biopsy showed no cirrhosis, stage 3 fibrosis (F3) was observed; fibrosis develops in patients with chronic liver damage due to viral hepatitis or a history of alcohol consumption. The lobular inflammation observed within the existing viral hepatitis and DILI was difficult to distinguish. These pathological findings indicated that DILI developed after the chronic liver injury.

\section{Discussion}

The details of this case show that the interaction between G/P therapy and host risk factors may induce serious adverse events. The excellent safety profile of $\mathrm{G} / \mathrm{P}$ has been demonstrated in several trials and studies (4-6). Although there have been reports of a transient elevation in serum bilirubin levels among patients treated with G/P $(10,11)$, severe liver injury associated with this treatment has not previously been reported.

NS3/4A protease inhibitors such as glecaprevir are pri- 


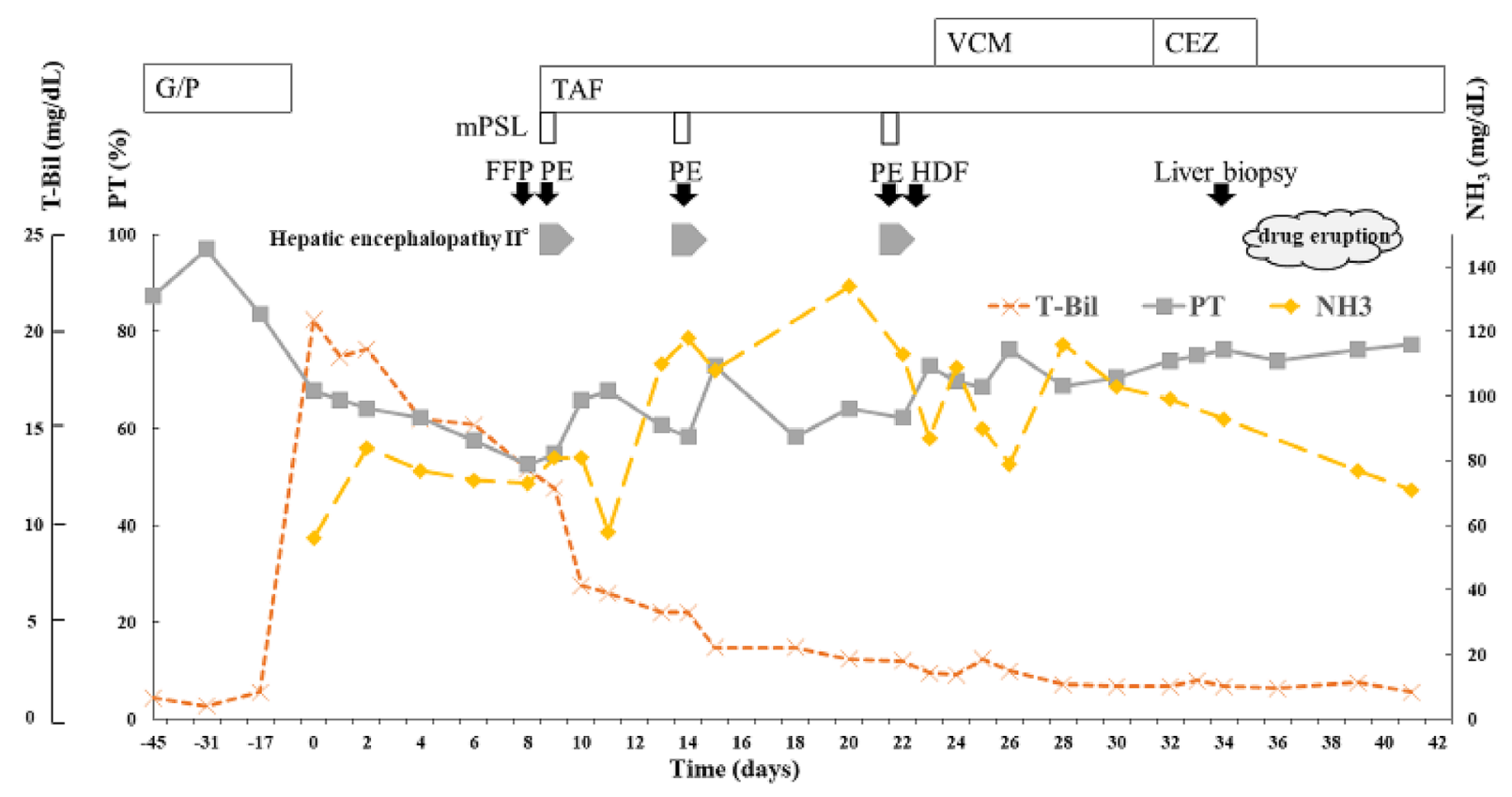

Figure 1. Clinical course. Days indicate days from admission. CEZ: cefazolin, FFP: fresh-frozen plasma, G/P: glecaprevir plus pibrentasvir, HDF: hemodiafiltration, mPSL: methylprednisolone, PE: plasma exchange, TAF: tenofovir alafenamide fumarate, VCM: vancomycin

Table 3. Serial Changes in Liver Function Tests, and Viral Markers during the Clinical Course.

\begin{tabular}{|c|c|c|c|c|c|c|c|c|c|c|c|}
\hline & $\begin{array}{l}\text { Diagnosis } \\
\text { of HCV }\end{array}$ & Base line & & & $\begin{array}{c}\text { Hospital } \\
\text { admission }\end{array}$ & H.E. & H.E. & H.E. & & & \\
\hline Date from admission & $\begin{array}{l}1 \text { year } \\
\text { ago }\end{array}$ & -45 & -31 & -17 & 0 & 9 & 14 & 22 & 23 & 33 & 41 \\
\hline Treatment contents & & $\begin{array}{c}\mathrm{G} / \mathrm{P} \\
\text { started }\end{array}$ & & & $\begin{array}{c}\mathrm{G} / \mathrm{P} \\
\text { stopped }\end{array}$ & $\begin{array}{c}\mathrm{PE} \\
\mathrm{mPSL}\end{array}$ & $\begin{array}{c}\mathrm{PE} \\
\mathrm{mPSL}\end{array}$ & $\begin{array}{c}\mathrm{PE} \\
\mathrm{mPSL}\end{array}$ & HDF & $\begin{array}{l}\text { Liver } \\
\text { biopsy }\end{array}$ & \\
\hline T.Bil (mg/dL) & 1.5 & 1.1 & 0.7 & 1.1 & 20.6 & 11.9 & 5.5 & 3 & 2.4 & 2 & 1.4 \\
\hline AST (IU/L) & 224 & 100 & 112 & 100 & 205 & 133 & 86 & 48 & 33 & 63 & 36 \\
\hline ALT (IU/L) & 107 & 51 & 63 & 51 & 65 & 56 & 45 & 27 & 22 & 30 & 15 \\
\hline ALP (IU/L) & 295 & 436 & 490 & 436 & 442 & 676 & 1,039 & 1,020 & 620 & 879 & 560 \\
\hline GGT (IU/L) & 659 & 125 & 83 & 125 & 124 & 138 & 207 & 274 & 131 & 141 & 84 \\
\hline $\mathrm{NH}_{3}(\mathrm{mg} / \mathrm{dL})$ & N.E. & N.E. & N.E. & N.E. & 73 & 81 & 118 & 113 & 87 & N.E. & 71 \\
\hline $\mathrm{PT}(\%)$ & 92.0 & 87.4 & 97.0 & 83.7 & 67.8 & 54.7 & 58.3 & 62.3 & 72.9 & 75.1 & 77.4 \\
\hline HCV-RNA (log IU/mL) & 5.8 & 1.4 & negative & N.E. & N.E. & N.E. & N.E. & N.E. & N.E. & negative & N.E. \\
\hline HBV-DNA (log IU/mL) & negative & negative & negative & N.E. & negative & N.E. & N.E. & N.E. & N.E. & negative & N.E. \\
\hline HBsAg (IU/mL) & 33.7 & 18.2 & 12.0 & N.E. & 7.8 & N.E. & N.E. & N.E. & N.E. & 11.5 & N.E. \\
\hline
\end{tabular}

AST: aspartate aminotransferase, ALT: alanine aminotransferase, ALP: alkaline phosphatase, GGT: gamma-glutamyl transpeptidase, G/P: glecaprevir plus pibrentasvir, HBsAg: hepatitis B surface antigen, HBV-DNA: hepatitis B virus DNA, HCV-RNA: hepatitis C virus RNA, HDF: hemodiafiltration, H.E.: hepatic encephalopathy, mPSL: methylprednisolone, N.E.: not examined, PE: plasma exchange, PT: prothrombin time

marily metabolized by $\mathrm{P} 4503 \mathrm{~A}$ and are contraindicated in decompensated cirrhosis due to significantly elevated protease inhibitor concentrations and an increased risk of liver toxicity (12-14). In terms of pharmacokinetics, the glecaprevir exposure was shown to be higher in patients with compensated cirrhosis than in those without cirrhosis, inducing possible hepatotoxicity $(15,16)$. Thus, variations in the safety and efficacy profile of G/P therapy in patients with compensated cirrhosis have been well-documented. In our patient, a liver biopsy indicated the absence of liver cirrhosis. NS5A inhibitors, such as pibrentasvir should also be considered to carry a risk of causing hepatoxicity. In the present patient, hyperbilirubinemia was assumed to be the result of drug- or metabolite-mediated inhibition of hepatobiliary transporters, but further research will be needed to determine the mechanism.

The reported incidence and severity of DILI varies among drugs (17-19), suggesting that drug properties play a role in determining the risk of DILI. On the other hand, only a small population of patients develop DILI, even after taking drugs with the potential to cause DILI, indicating that host factors play a major role in DILI development. Known host 
(a)

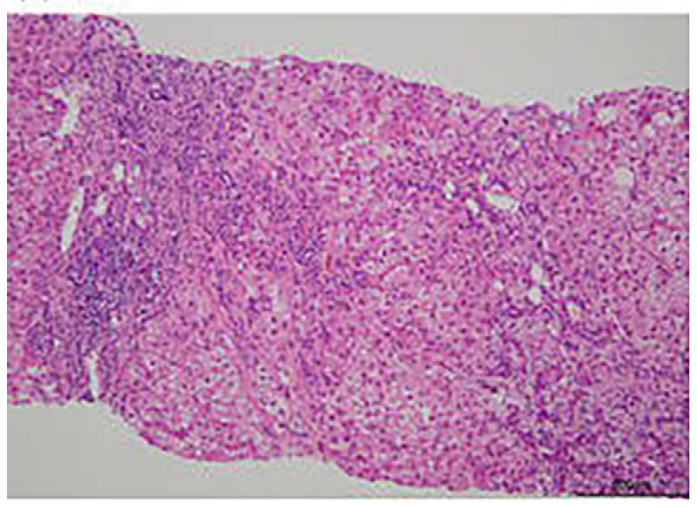

(c)

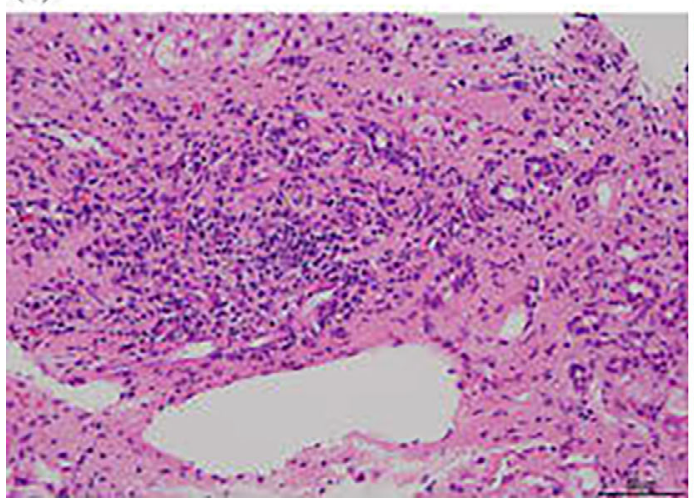

(b)

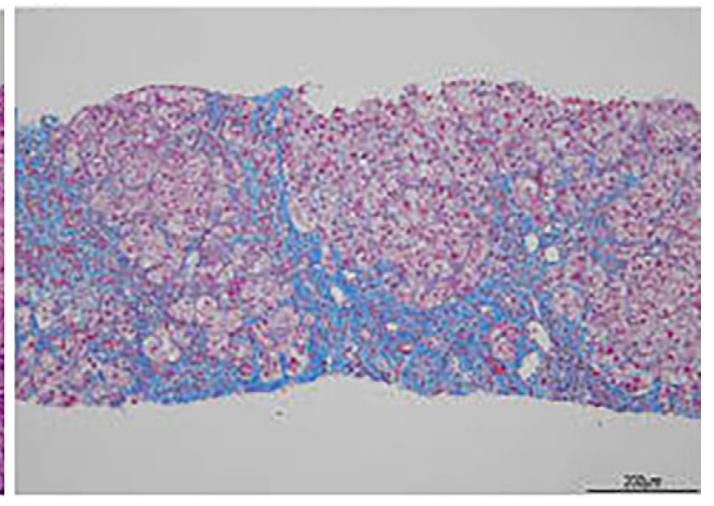

(d)

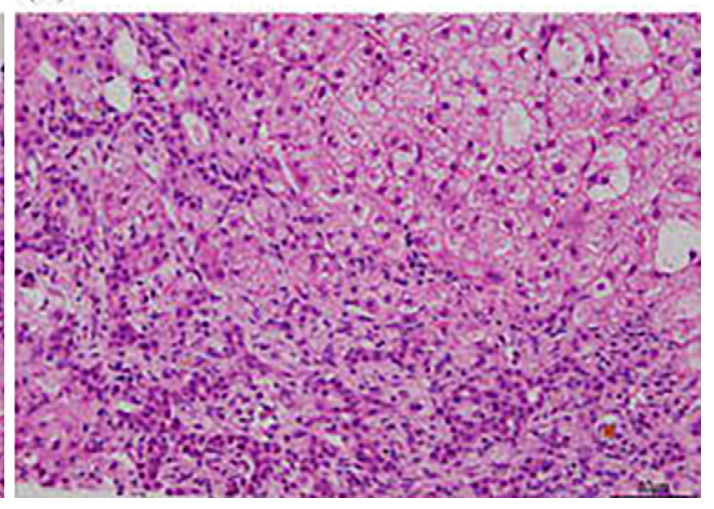

Figure 2. Histological findings from the liver biopsy specimen. (a) Portal inflammation with slight interface hepatitis [Hematoxylin and Eosin $(H \& E)$ staining; magnification: $\times 100]$. (b) Fibrosis with portal-to-portal bridging (Masson's trichrome staining; magnification: $\times 100)$. (c) Mixed lymphocyte and plasma cell infiltration and rare eosinophils in the portal area with ductular proliferation (H\&E staining; magnification: $\times 200)$. (d) Mild cholestasis and ballooning hepatocytes $($ H\&E staining; magnification: $\times 200)$.

risk factors include an increased age, female sex, presence of underlying liver disease, and heavy alcohol intake; in addition, several genetic variants in the human leukocyte antigen (HLA) regions have been identified as risk factors for idiosyncratic DILI (7). Heavy alcohol consumption is a risk factor for DILI because the direct hepatotoxicity induced by ethanol and indirect hepatotoxicity induced by its metabolite (acetaldehyde) result in hepatocellular damage (20). Our patient had a habit of heavy alcohol consumption six months earlier, although it had not caused the present DILI. Among patients receiving antiviral treatment for viral hepatitis, our patient had no particular risk factor for DILI.

$\mathrm{HBV}$ reactivation during or after DAA therapy is frequent among $\mathrm{HBV} / \mathrm{HCV}$-coinfected patients (21). In our case, $\mathrm{HBV}$ reactivation was excluded from the potential causes of liver injury, as the level of serum HBV DNA remained negative, and the level of HBs-Ag was low during DAA therapy. Since it was necessary to use an immunosuppressive drug to manage anaphylaxis during the clinical course, TAF treatment was initiated to prevent $\mathrm{HBV}$ reactivation. Regarding the relationship between $\mathrm{HBV} / \mathrm{HCV}$-coinfection and DILI, the inflammation and altered cytokine milieu caused by a chronic viral disease may influence drug hepa- totoxicity (22).

Our patient also had a history of drug allergy against two drugs other than the G/P. Laboratory findings related to the presence of allergies included a negative result of DLST for $\mathrm{G} / \mathrm{P}$, and low levels of serum eosinophils. According to previous reports, DLST was positive in $48 \%$ of patient with DILI, and eosinophilia was greater than $6 \%$ in $27 \%$ of patients with DILI (23). Thus, the sensitivity of these factors is not high, and these findings are useless for diagnosing drug allergy.

Factors causing immune allergic response are involved in the mechanism underlying cellular injury in idiosyncratic $\operatorname{DILI}(7,22)$. For example, allergies are caused by excessively strong immune functions, and patients with an allergic constitution are often more sensitive to drugs and show a higher incidence of DILI than patients without allergic constitution (20). At present, a history of drug allergy is not listed as a risk factor for DILI (7, 7-19). Although the overall incidence of drug allergy is unknown, it accounts for 1$2 \%$ of all admissions and 3-5\% of hospitalized patients (24). According to a report by the Drug-induced Liver Injury Network, patients with DILI frequently $(>40 \%)$ have a history of drug allergy (19). Therefore, the history of drug allergy 
may have been a host risk factor for DILI in this case.

DILI is a leading cause of acute liver failure $(7,9,17,19$, 23 ), with $1 \%$ of cases being subjected to PE (23). In Japan, artificial liver support, consisting of $\mathrm{PE}$ and $\mathrm{HDF}$, is performed as treatment for patients with acute liver failure, especially in those with hepatic encephalopathy to stabilize the patient's condition until recovery of the native liver or performance of liver transplantation $(9,25)$. Disaccharides and the nonabsorbable antibiotic rifaximin are also recommended for the treatment of hepatic encephalopathy for patients with cirrhosis (26). In our patient, the PT did not meet the criteria for acute liver failure (9); however, he suffered from severe liver injury, hyperbilirubinemia, deterioration of PT over time, and the development of grade II hepatic encephalopathy. The results of a liver biopsy showed sever liver injury with jaundice, mixed lymphocyte and plasma cell infiltration in the portal vein area, ductular proliferation, ballooning hepatocytes, and cholestasis. Oral treatment of lactulose and rifaximin for hepatic encephalopathy, and artificial liver support were useful for maintaining the minimal liver function required to sustain the life of this patient.

Recently, it was reported that the recovery rate from hepatic encephalopathy is higher in patients with PE and HDF than in those with PE alone (25). This is because HDF removes low- to middle-sized molecules, including ammonia, decreasing the side-effects of PE. In the present case, hepatic encephalopathy was not observed after the combined use of PE and HDF. Although anaphylaxis and catheter infection occurred as a complication of plasmapheresis, with appropriate treatment, the patient eventually recovered from his severe liver injury.

In conclusion, we encountered a case of a severe liver injury caused by G/P therapy. Host factors should be considered in order to prevent DILI during treatment with this regimen.

The authors state that they have no Conflict of Interest (COI).

\section{Acknowledgement}

The authors thank Drs. S. Hosoi and Y. Okumura for their cooperation in the management of the reported case.

\section{References}

1. Ghany MG, Strader DB, Thomas DL, Seeff LB; American Association for the Study of Liver Disease. Diagnosis, management, and treatment of hepatitis C: an update. Hepatology 49: 13351374, 2009.

2. Falade-Nwulia O, Suarez-Cuervo C, Nelson DR, Fried MW, Segal JB, Sulkowski MS. Oral direct-acting agent therapy for hepatitis C virus infection: a systematic review. Ann Intern Med 166: 637648, 2017.

3. Zeuzem S, Foster GR, Wang S, et al. Glecaprevir-Pibrentasvir for 8 or 12 weeks in HCV genotype 1 or 3 infection. N Engl J Med 378: 354-369, 2018.

4. Forns X, Lee SS, Valdes J, et al. Glecaprevir plus pibrentasvir for chronic hepatitis $\mathrm{C}$ virus genotype 1, 2, 4, 5, or 6 infection in adults with compensated cirrhosis (EXPEDITION-1): a single-arm, open-label, multicentre Phase 3 trial. Lancet Infect Dis 17: 1062-
1068, 2017.

5. Toyoda H, Chayama K, Suzuki F, et al. Efficacy and safety of glecaprevir/pibrentasvir in Japanese patients with chronic genotype 2 hepatitis $C$ virus infection. Hepatology 67: 505-513, 2018.

6. Kumada H, Watanabe T, Suzuki F, et al. Efficacy and safety of glecaprevir/pibrentasvir in $\mathrm{HCV}$-infected Japanese patients with prior DAA experience, sever renal impairment, or genotype 3 infection. J Gastroenterol 53: 566-575, 2018.

7. Chen M, Suzuki A, Borlak J, Andrade RJ, Lucena MI. Druginduced liver injury: interactions between drug properties and host factors. J Hepatol 63: 503-514, 2015.

8. Takikawa K, Onji M, Takamori Y, et al. Proposal of diagnostic criteria of drug induced hepatic injury in DDW-J2004 workshop. Kanzo 46: 85-90, 2005 (in Japanese).

9. Sugawara K, Nakayama N, Mochida S. Acute liver failure in Japan: definition, classification, and prediction of the outcome. J Gastroenterol 47: 849-861, 2012.

10. Hammami MB, Aboushaar R, Alsabbagh E. Glecaprevir/ pibrentasvir-associated acute liver injury in non-cirrhotic, chronic $\mathrm{HCV}$ infection without HBV co-infection. BMJ Case Rep 12: e231744, 2019.

11. Yoon JH, Kim SM, Kang G, Kim HJ, Jun CH, Choi SKl. A case report of glecaprevir/pibrentasvir-induced severe hyperbilirubinemia in a patient with compensated liver cirrhosis. Medicine (Baltimore) 98: e17343, 2019.

12. European Association for the Study of the Liver. EASL recommendations on treatment of hepatitis C 2018. J Hepatol 69: 461511, 2018.

13. Hepatitis C Guideline 2018 Update: AASLD-IDSA recommendations for testing, managing, and treating Hepatitis $\mathrm{C}$ virus infection. Clin Infect Dis 67: 1477-1492, 2018.

14. Drafting Committee for Hepatitis Management Guidelines the Japan Society of Hepatology. Japan Society of Hepatology guidelines for the management of hepatitis C virus infection; 2019 update. Hepatol Res 50: 791-816, 2020.

15. Gane E, Poordad F, Zadeikis N, et al. Safety and pharmacokinetics of glecapirevir/pibrentasvir in adults with chronic genotype 1-6 hepatitis $\mathrm{C}$ virus infections and compensated liver disease. Clin Infect Dis 69: 1657-1664, 2019.

16. Suleiman AA, Lin CW, Liu W, et al. Population pharmacokinetics of glecapirevir/pibrentasvir in HCV-infected Japanese subjects in phase 3 CERTAIN-1 and CERTAIN-2 trials. J Clin Pharmacol 60: 331-339, 2020.

17. Bjornsson ES, Bergmann OM, Bjornsson HK, Kvaran RB, Olafsson S. Incidence, presentation, and outcomes in patients with drug-induced liver injury in the general population of Iceland. Gastroenterology 144: 1419-1425, 2013.

18. Shin J, Hunt CM, Suzuki A, Papay JI, Beach KJ, Cheetham TC. Characterizing phenotypes and outcomes of drug-associated liver injury using electronic medical report data. Pharmacoepidemiol Drug Saf 22: 190-198, 2013.

19. Chalasani N, Bonkovsky HL, Fontana R, et al. Features and outcomes of 899 patients with drug-induced liver injury: The DILIN prospective study. Gastroenterology 148: 1340-1352, 2015.

20. Lu RJ, Zhang Y, Tang FL, et al. Clinical characteristics of druginduced liver injury and related risk factors. Exp Ther Med 12: 2606-2616, 2016.

21. Mucke MM, Backus LI, Mucke VT, et al. Hepatitis B reactivation during direct-acting antiviral therapy for hepatitis $\mathrm{C}$ : a systematic review and meta-analysis. Lancet Gastroenterol Hepatol 3: 172$180,2018$.

22. Kaplowitz N. Idiosyncratic drug hepatotoxicity. Nat Rev Drug 4: 489-499, 2005.

23. Aiso M, Takikawa H, Tsuji K, et al. Analysis of 307 cases with drug-induced liver injury between 2010 and 2018 in Japan. Hepatol Res 49: 105-110, 2019. 
24. Thong BY, Tan TC. Epidemiology and risk factors for drug allergy. Br J Clin Pharmacol 71: 684-700, 2011.

25. Fujiwara K, Abe R, Yasui S, Yokosuka O, Kato N, Oda S. High recovery rate of consciousness by high-volume filtrate hemodiafiltration for fulminant hepatitis. Hepatol Res 49: 224-231, 2019.

26. Fukui H, Saito H, Ueno Y, et al. Evidence-based clinical practice guidelines for liver cirrhosis 2015. J Gastroenterol 51: 629-650,
2016.

The Internal Medicine is an Open Access journal distributed under the Creative Commons Attribution-NonCommercial-NoDerivatives 4.0 International License. To view the details of this license, please visit (https://creativecommons.org/licenses/ by-nc-nd/4.0/).

(C) 2021 The Japanese Society of Internal Medicine Intern Med 60: 2437-2443, 2021 